\title{
Interdisciplinariedad en gerontología. La calidad de vida como criterio integrador de la interdisciplina
}

\author{
Interdiscipline in gerontology. \\ The quality of life like integration \\ criterio of the Interdisciplinary
}

\author{
José Enrique Gómez Álvarez*
}

\section{Resumen}

El artículo presenta un análisis del término multidisciplina e interdisciplina en Gerontología. Para lograr lo anterior se presenta una analogía con el uso del término interdisciplina en Bioética. Se parte de la consideración de la ética como unificador metodológico de la Bioética que permite pasar de ser una multidisciplina a una interdisciplina. De modo análogo, se aplica ese sentido al término gerontología, y se propone así el uso del término «calidad de vida activa debida» como eje ético que oriente la gerontología.

Palabras clave: interdisciplina, epistemología, calidad de vida, envejecimiento.

\footnotetext{
* Doctor en Filosofía por la Universidad de Navarra. Maestro en Gerontología Social. Profesor e investigador del CISAV.

Correo electrónico: jegomezalvarez@yahoo.com

Recibido el 04 de septiembre de 2019. Entregado el 30 de septiembre de 2019.
} 


\section{Introducción: la bioética cómo ética aplicada, punto de partida de reflexión sobre el método}

Uno de los problemas de la interdisciplina denominada «Bioética» es, en realidad, establecer su estatuto epistémico y su alcance, y es un tema no concluido en tal campo. El término nos permite dar un marco para entender los problemas con otras interdisciplinas, en particular con el de "gerontología». Ana Marta González resume de manera adecuada la posición del estatuto epistemológico de la Bioética. La Dra. González expone sus razones para descartar a la Bioética como una interdisciplina (he subrayado lo que considero más importante de resaltar):

Los partidarios de constituir a la Bioética en una ciencia autónoma pueden alegar a su favor el hecho innegable de que ha generado, en muy pocas décadas, una reflexión ingente y específica que, además de requerir la aplicación de los principios éticos a una materia muy concreta, exige internamente la adopción de una perspectiva multidisciplinar a la hora de enfrentar sus problemas específicos; aunque esta postura es defendible desde un punto de vista práctico, considero que por sí sola no autoriza a conceder a la Bioética un estatuto epistemológico diverso al de la Ética. Pues, de una parte la concreción de su materia no bace de ella una ciencia diversa, ya que también aqui se trata de acciones bumanas (por mucho que la materia de estas acciones se circunscriba a un ámbito determinado) y de otra, su mismo carácter interdisciplinar -que es basta cierto punto lo más novedoso de la Bioética- no constituye tampoco un motivo suficiente para constituirla en una ciencia independiente. Después de todo, la misma interdisciplinariedad podría verse como una ampliación sistemática de la deliberación que ha de preceder a toda decisión éticamente aceptable, cuya aceptabilidad, en todo caso, corresponde examinar a la ética. ${ }^{1}$

El pasaje anterior nos remite a dos problemas. Uno es qué disciplina o qué elemento cognitivo sirve de enlace o de conexión entre las disciplinas. En el campo de la Bioética, sin duda es la pregunta filosófica del deber ser la que de algún modo enlaza las disciplinas 
participantes. La ética, en pocas palabras, o la pregunta ética que se plantea sirve de conexión entre el Derecho, la Medicina, el Trabajo Social, la Enfermería, entre otras, cuando intervienen en la decisión bioética. La Dra. González señala un tema crucial: el objeto señala el método. El objeto de la Bioética, los actos humanos, son tema propio de la ética al considerar su objeto formal; es decir, en cuanto buenos o malos. Lo anterior apunta a que la Bioética a lo sumo es un área de la ética, como podría ser la ética social, por ejemplo. Llevando esto a la gerontología, la pregunta sobre su carácter interdisciplinario se formula así: ¿Qué objeto tiene la gerontología que lo convierte en algo interdisciplinario? La gerontología estudia la vejez. En eso, su objeto material no se diferencia de la geriatría, por ejemplo. La clave está en el objeto formal. Mencionaré más adelante esta cuestión.

Otro elemento digno de resaltar del texto de González es la utilización indistinta de dos términos: multidisciplinar e interdisciplinar. Cómo veremos en la siguiente sección, no son equivalentes y eso dará pauta para discutir el sentido de la gerontología.

\section{La gerontología como interdisciplina}

De acuerdo con lo visto en la introducción, la interdisciplina o multidisciplinario pueden entenderse como colaboración de grupos de trabajo con una meta común, como sugiere el texto de González. Así, algunos autores comprenden la noción de «interdisciplina» en la atención de la vejez. Sin embargo, otros autores hacen una distinción en el cuidado de pacientes ancianos.

Equipo multidisciplinario es un grupo de diferentes profesionales que trabajan en un área común de forma independiente, valoran al enfermo por separado e interactúan entre ellos de manera informal; o sea el enfoque asistencial se da en paralelo, sin integrar la asistencia en un plan global ni establecer prioridades. 
Equipo interdisciplinario es el constituido por diferentes profesionales que trabajan en un área común de forma interdependiente e interactúan entre ellos de manera formal e informal. Pueden valorar al enfermo por separado pero intercambian la información de una forma sistemática, comparten una metodología de trabajo y laboran juntos para conseguir unos objetivos conjuntos, colaborando entre ellos en la planificación y puesta en marcha de un plan de tratamiento y cuidados. ${ }^{2}$

Y nos remite así a que a veces en el campo de la Bioética se trabaja multidisciplinariamente, buscando resolver o mejor recolectar las opiniones de los profesionistas y dar una respuesta a una consulta ética. Entendida así, la Bioética no es interdisciplina, y sólo es una ética aplicada. Pero como interdisciplina, la respuesta ética o la pregunta filosófica «¿Debo hacer X porque puedo hacerlo?» nos remite a una pregunta guía común y a buscar entre todos responder una misma pregunta, claro desde la disciplina de cada área, pero unificada por el cuestionamiento ético. Así, la Bioética, aunque estudie los actos humanos en cuanto buenos o malos, y en eso no hay diferencia con la ética, la respuesta tiene implicaciones que rebasan solo la moralidad o, mejor dicho, la moralidad invade otras áreas que ya no son especialidad de la ética, como puede ser el derecho, lo que implica que la respuesta ética ya no puede darse sólo desde la reflexión filosófica. Se requiere la participación de otra área para completar la respuesta. Es verdad que seguimos en el campo ético, pero requerimos de otra disciplina para, por decirlo metafóricamente, «colorear» la respuesta con un dato adicional, como puede ser la legalidad del acto moral. La ética ya es la respuesta buscada en el Derecho o en la Medicina o en la disciplina que participa.

En el caso de la gerontología, ¿cuál puede ser la pregunta unificadora o el concepto unificador que permita superar la mera consulta entre saberes diversos? La gerontología tiene un objeto común con otras disciplinas, el envejecimiento como ya se dijo, pero, ¿qué es lo específico de la gerontología? A diferencia de la ética no 
es una ciencia única con un objeto formal específico. La gerontología hace alusión el estudio del envejecimiento en cuanto todos los elementos que conlleva. ${ }^{3}$ La gerontología, a diferencia de la geriatría, que estudia en gran medida lo patológico en el envejecimiento, busca entenderlo siempre como un todo complejo, de ahí su carácter cuando menos multidisciplinario o de consulta entre disciplinas.

Pero propongo que el trabajo interdisciplinar en la gerontología es colaboración integrada por valores y metas comunes. ${ }^{4}$ No se renuncia a la metodología propia de cada área, pero sí debe existir un hilo conductor, un concepto guía que busca responderse desde las disciplinas y posteriormente se conecta con el problema a resolver. La interdisciplina es trabajar con un marco teórico común, una noción epistemológica compartida. En el caso de la Bioética puede ser el valor de la vida humana.

La gerontología, de modo análogo, es planteada a veces como una multidisciplina, a veces como una interdisciplina. Por ejemplo, señala una autora respecto al plan de estudios de licenciatura en Gerontología:

Una característica de la licenciatura en Gerontología es su multidisciplinariedad, por lo que tiene un amplio mapa curricular, sumergido en distintas materias y ramas del conocimiento que deben enfocarse hacia el proceso de envejecimiento en su totalidad; lo que se hace a través de una pluralidad de profesores, muchos contratados por asignatura que sólo imparten una o dos materias, sin tiempo de involucrarse con la enseñanza de una gerontología, que requiere el desarrollo de conocimiento específico sobre vejez. ${ }^{5}$

De momento conviene resaltar el «deben enfocarse hacia el proceso de envejecimiento en su totalidad». ¿Qué puede significar lo anterior? ¿Qué usos le podemos atribuir al término gerontológico? Primero esbozaré un par de ejemplos de uso, y posteriormente compararemos con algunos resultados muy interesantes del artículo citado de cómo conciben los propios profesores esta interdisciplina. La gerontología estudia todas las causas del envejecimiento. 
Para la planeación de intervenciones debemos tener una idea de qué intervenimos. Así se ha planteado si la gerontología es el proceso entero de la vida o, si al delimitarla a un periodo de la misma, digamos los 60 años en adelante, cómo lo justificaríamos. Las causas del envejecimiento se dan desde el mismo momento que nacemos. ${ }^{6}$ Es sabido que existen diversas teorías del proceso; por ejemplo, las teorías orgánicas, las fisiológicas y las genéticas. ${ }^{7}$ De cualquier forma, envejecemos en diferente grado y desde jóvenes. Tomando en cuenta lo anterior, la gerontología prevé o plantea el papel del envejecimiento desde que nacemos. Así, el gerontólogo aunque realice intervenciones en las edades «clásicas» de la vejez, asociadas a la jubilación laboral, por ejemplo, en realidad debería considerar su planeación desde el nacimiento mismo hasta la muerte. Por ejemplo, el gerontólogo debe tener intervenciones hacia atrás en el tiempo. Se debe prever una vejez saludable desde niños y en la misma juventud. Un plan de intervención, por ejemplo, en diabetes debe incluir que se incida no sólo hacia la población objetivo, sino hacia atrás aunque sean jóvenes. Se explica así que las campañas de prevención se solapan entre todas las edades. Un modo de resumirlo es diciendo que:

Se debe poder trabajar desde la gerontología con un equipo interdisciplinario, en donde cada disciplina sabe de la otra lo que necesita y le bace falta para poder intervenir mejor. No es que una disciplina trata de hacer lo que le corresponde a otra, sino que se tienen en consideración a las demás disciplinas que intervienen para poder desarrollar un trabajo en todos los aspectos. ${ }^{8}$

Otro modo de resumir la propuesta es que la intervención multidisciplinaria consiste en que cada disciplina aporte su opinión acerca de un tema o pregunta desde su disciplina, sin pretender resolver lo ético. La interdisciplina es más que eso, es contestar desde la ética y cada disciplina contesta como una persona que resuelve desde su disciplina, pero contestando éticamente. El abogado contesta su solución ética. Toma de la filosofía lo que necesita para 
responder como abogado el problema ético en el caso de la Bioética. La gerontología es el cuidado en la vejez; sugiero utilizar o que se pueda utilizar la categoría del cuidado como eje articulador de todos los participantes.

El gerontólogo tiene un papel práctico humanista. Se debe dedicar al cuidado de los viejos en todas sus facetas. «Cuidado» es asociado con frecuencia a fragilidad presente y preservar lo que se tiene para no caer en aquélla. El gerontólogo, así, debe evitar o disminuir la fragilidad propia de la vejez, así como crear condiciones para que no se dé la misma si esto es posible.

Regresando al estudio de Angélica María Razo, éste nos muestra que existe una variación importante en la definición o descripción de la gerontología, dependiendo de la formación profesional y del acercamiento al ámbito académico. Señala en las conclusiones un dato interesante:

En cuanto al primer concepto, para el total de profesores, la gerontología es una ciencia que se ocupa del envejecimiento como objeto de estudio primordialmente y con una fuerte concepción integradora, lo cual es congruente con la perspectiva amplia e integral que se espera tengan de acuerdo con la misión de la carrera y el plan curricular, puesto que en teoría han acumulado información suficiente para objetivar y naturalizar el concepto.

Sin embargo, llama la atención que mientras en la visión de la licenciatura se contempla que los gerontólogos sean profesionistas "con un amplio sentido humanista y científico», la palabra definidora «bumanista» ocupa el último lugar del núcleo central de su campo de la representación, con la quinta parte del peso semántico respecto a la de mayor peso semántico que es la palabra «ciencia». ${ }^{9}$

Dicho de otro modo, se debe buscar un equilibrio dentro de la gerontología, con su visión humanista del cuidado de las personas ancianas junto con el carácter científico de la interdisciplina. En otros términos, la gerontología no debe quedar en buenas intenciones en el cuidado de las personas e ir más allá con una metodolo- 
gía científica en donde se planifiquen, ejecuten y evalúen las intervenciones con los estándares científicos de las disciplinas involucradas. Pero por otra parte, el cuidar, cómo dato axiológico, la categoría aplicable a las disciplinas de la salud es esencial. ${ }^{10}$ Subrayé en la cita ese aspecto de la misión de la carrera profesional de gerontólogo; esa misión es una categoría ética y que, espero mostrar un poco más adelante, es el criterio unificador para su carácter interdisciplinario. La gerontología ha replanteado la categoría de la vejez de modo que se plantea como interdisciplina desde su origen, ya que:

La reconfiguración de las fases de la vida y de la salud como un proceso de vida tiene consecuencias en las formas en las que se clasifican la edad y la vejez, al menos en tres aspectos. En primer lugar, la categoría de edad perdió sus cualidades causales, lo que significó que la trayectoria de vida varió su estándar ordinal... El envejecimiento categoriza entonces el proceso en lugar del número... En segundo lugar, cuando la edad es sustituida por el envejecimiento, surgen preguntas en torno a la clasificación de la vejez. ¿Cuándo comienza la vejez? ¿Puede clasificarse utilizando una cifra? En tercer lugar, las críticas surgidas en contra de la clasificación de la «vejez» apuntan también hacia la forma en que la vejez se ha utilizado como categoría social, clasificando el comportamiento y la identidad de los individuos en esta categoría. Ser mayor -si esto significa tener más de 65 años, estar débil, necesitar atención, o recibir una pensión estatal- sugiere una identidad y un comportamiento específicos. Pero a menudo, la gente mayor no se identifica con su edad..., ni tampoco se comporta siempre tal y como se espera de ellos». ${ }^{11}$

Así, la vejez es un constructo conceptual multidimensional no identificable fácilmente con una conducta esperable o una edad exacta. Entonces, el entender y manejar el envejecimiento, que como decíamos se da desde el momento mismo en que nacemos, implica una proyección del tipo de vida que se espera de uno mis- 
mo y del orden social existente. Entender la vejez es replantearse la misma incluso desde su misma localización espacial; es decir, existe una geografía del envejecimiento. ${ }^{12}$ Debido a que el «cuidado para evitar la fragilidad», resumiendo lo anteriormente dicho, implica aspectos de salud, infraestructura urbana, ecología, nutrición y un largo etcétera. ${ }^{13}$ La categoría del cuidado para evitar la fragilidad del desarrollo podría agregarse, es interdisciplinaria de origen.

La manera de afrontar la vejez, desde su misma conceptualización, es interdisciplinaria. Otro concepto tradicional que completa el carácter interdisciplinario es el de envejecimiento activo. El mismo, de nuevo, se aplicaría a todo el proceso de cualquier ciclo vital. Se hace énfasis en el aprovechamiento de cualquier oportunidad de desarrollo, buscando así el bienestar en todos los ámbitos con el «objetivo de extender la calidad y esperanza de vida a edades avanzadas». ${ }^{14}$ De nuevo esto se resuelva desde distintas disciplinas. El envejecimiento activo como tal es un ideal de la razón práctica que asumen los participantes cuando se plantea intervenir en la vejez.

Pero lo que interesa resaltar aquí es que podemos y debemos concebirla como una interdisciplina, ya que busca atender un deber ético, el proporcionar calidad a la vejez por medio de una planeación del cuidado. Esa calidad de vida implica niveles o aspectos diferentes: clínicos, ambientales, relaciones sociales que modifican la expectativa sobre la propia vejez y la ajena. Esa expectativa se convierte en un deber o, dicho de otro modo, al pensar las condiciones que garanticen un envejecimiento activo se me descubre como una serie de bienes debidos. Esos bienes debidos nos llevan a que en toda intervención gerontológica exista una pregunta general que todas las disciplinas involucradas deben contestar: ¿cómo cuidar a la persona para evitar la fragilidad inherente al proceso irreversible del desarrollo humano? Esa pregunta común es la que permite unificar metas e interrelacionar métodos para resolver la cuestión de qué ver aquí y ahora con determinada situación que queremos modificar de la persona anciana. 


\section{Calidad de vida como criterio ético de la interdisciplina en gerontología}

Propongo que, de un modo semejante a la Bioética, el eje conductor o aglutinador de la gerontología como interdisciplina sea la noción de «calidad de vida debida». Pero la noción de calidad de vida propongo entenderla como un concepto ético. Dicho en otros términos, como un concepto aspiracional, un deber ser de la atención al anciano. «Calidad de vida» se convierte así en cómo debe ser la vida humana en cuanto las condiciones de posibilidad de recrearse continuamente. La calidad de vida es más amplia que las situaciones de salud y enfermedad. La calidad de vida implica aspectos sociales como la disposición de tiempo libre, ingresos, bienes materiales y factores como seguridad personal, vivienda. Es claro, asimismo, que hay factores subjetivos difíciles de determinar: estilo de vida y satisfacción por las actividades que se realizan. ${ }^{15}$

Podemos unir este concepto de calidad de vida con otro muy utilizado en los temas de gerontología y que ya indicamos en el epígrafe anterior: «envejecimiento activo». Veíamos que la vejez como concepto etiquetador se modifica con la noción de actividad. Si se entiende la vejez más bien como otro ciclo más de actividad personal y se rompen las expectativas de conducta de las personas ancianas, la vejez deja de ser de personas «decadentes» y se mantienen ideales y formas de vida de otras etapas. ${ }^{16}$ De ahí que el término propuesto para entender el trabajo interdisciplinario sea «calidad de vida activa debida».

\section{A manera de conclusión}

No hay acuerdo definitivo sobre el carácter interdisciplinario y el multidisciplinario. La propuesta de este artículo es que hay una diferencia entre ambos. El equipo interdisciplinario tanto en gerontología como en bioética se da cuando se mantienen los métodos 
propios de las disciplinas pero se engarzan en responder la misma problemática ética. El concepto ético se convierte en unificador de las mismas al contestar desde la ética completada por el conocimiento de la disciplina no ética. En cambio, multidisciplinar, propongo, hace referencia a la mera consulta sobre un dato del problema, sin pretender responderlo éticamente.

A partir de las reflexiones anteriores puede construirse una propuesta de metodología de análisis de casos. Eso se constituiría en otro tema de investigación posterior.

\section{Referencias bibliográficas}

Álvarez, Virginia. Palumbo, Raquel. Rodríguez, Cecilia. Salveraglio, Inés. SilVEIRA, MÓNICA. (2018). Trabajo con personas adultas mayores. Comisión Sectorial de Educación Permanente (CSEP). Universidad de la República de Uruguay.

Araújo, Sarah Nilkece Mesquita. Santiago, Roberta Fortes. Barbosa, Cristhiano NeIVA Santos. Figueiredo, PIOVesan Sylvia. Aria do LiVRamento Fortes. Andrade, Elaine Maria leite Rangel. Nery, Inez Sampaio. Tecnologías orientadas al cuidado del anciano en los servicios de salud: una revisión integradora. Enfermería global 2017; 46.

CABERO, LAURA ADRIANA. Una reflexión sobre la intervención social en la expresión actual de la vejez. Margen. 2017; 87. Consultado el 4 de diciembre de 2018. Disponible en: https://www.margen.org/suscri/margen87/cabero_87.pdf

De LEÓn, Álvaro. (2013). El abordaje interdisciplinar en la vejez y el envejecimiento con riesgo social.» Un estudio de caso en la Cátedra de Geriatría del Hospital de Clínicas (tesis). Montevideo: Universidad de la República de Uruguay. Consultado el 3 de diciembre de 2018. Disponible en: file:///D:/2018/Gerontología $\% 20$ e $\% 20$ interdisciplina/El\%20abordaje $\% 20$ interdisciplinar $\% 20$ en $\% 201 a \%$ 20vejez.pdf

GonzÁlez, AnA MARTHA. 2001. Claves éticas para la Bioética. Cuadernos de Bioética. $3^{a}$, p. 305. Consultado el 5 de diciembre de 2018. Disponible en: http:// aebioetica.org/revistas/2001/3/46/305.pdf

Castanedo Granda, Juan Francisco. GonzÁlez Moro, Alina María. ¿Es necesaria la interdisciplinaridad en la atención gerontológica en el nivel primario? Geo info. 2015; 10(2).

Dutra lemos. Naiara de fatima. Andrade Tobias, Mónica. Cristiane LUIZ, CristIANE. MARIELA BESSE, MARIELA. (2012). Interdisciplinaridade, saúde e gerontologia: Articulando saberes. Revista Equilibrio Corporal e Saúde. 2012; 4(1): 3-8.

Medicina y Ética - Octubre-Diciembre 2019 - Vol. 30 - Núm. 4 
RAZO, ANGÉLICA MARÍA. (2014). El quehacer del gerontólogo: representaciones sociales de los profesores de la licenciatura en Gerontología de la UNEVE (México). Revista Internacional de Estudios sobre Sistemas Educativos/ International Journal of Studies in Educational Systems. 2014; 2(4): p.54.

BORRERO CABAL, ALFONSO. En busca de la interdisciplinariedad: interdisciplinariedad y gerontología. (Consultado el 2 de diciembre de 2018). Disponible en: https:/ /www.gerontologia.org/portal/information/showlnformation. .php?idinfo=862

BRAILOVSKY, S. RodrígueZ, R. (2004). Teorías sobre el envejecimiento. En Geriatría. Mc Graw Hill, México. Pp. 25-27.

LASSEN JUUL, ASKE. Biopolíticas de la vejez. Cómo el conocimiento sobre el envejecimiento forma políticas de envejecimiento active. Sociología Histórica 5/2015: 342-343.

MORALES VIRGEN, JUAN JosÉ. (2004). Retos de la investigación gerontológica. En Geriatría. Mc Garw-Hill, México.

SÁNCHEZ GonZÁLEZ, DIEGo. (2015). Ambiente físico-social y envejecimiento de la población desde la gerontología ambiental y geografía. Implicaciones socioespaciales en América Latina. Revista de Geografía Norte Grande, 60: 97-114.

OMS (2001). Campaña de la OMS por un envejecimiento activo, p. 2 (Consultado el 2 de diciembre de 2018). Disponible en: https://www.who.int/ageing/publications/alc_elmanual.pdf?ua=1

Pietro Ramos, Osvaldo. (1999). Gerontología y Geriatría. Breve resumen histórico. Resumed 12 (2) 51-54. (Consultado el 3 e diciembre de 2018). Disponible en: http://www.bvs.sld.cu/revistas/res/vol12_2_99/res01299.pdf

Rodríguez García, Rosalía. Mendoza Martínez, Lilia. (2004). Calidad de Vida. En Geriatría. Mc Graw-Hill, México.

\section{Notas bibliográficas}

1 GonzÁlez, ANA MARTHA, 2001: 305.

${ }^{2}$ Castanedo Granda, Juan Francisco. GonzÁlez Moro, Alina María. ¿Es necesaria la interdisciplinaridad en la atención gerontológica en el nivel primario? Geo info. 2015; 10(2).

${ }^{3}$ Cf. Pietro Ramos, Osvaldo. (1999). Gerontología y Geriatría. Breve resumen histórico. Resumed 12 (2) 51-54. Consultado el 3 e diciembre de 2018. Disponible en: http://www.bvs.sld.cu/revistas/res/vol12_2_99/res01299.pdf

4 Dutra lemos. Naiara de fatima. Andrade Tobias, Mónica. Cristiane luiz, CRistiane. MARIELA Besse, MARIELA. Interdisciplinaridade, saúde e gerontologia: Articulando sabers. Revista Equilíbrio Corporal e Saúde. 2012; 4(1): 3-8.

${ }^{5}$ El quehacer del gerontólogo: representaciones sociales de los profesores de la licenciatura en Gerontología de la UNEVE (México). Revista Internacional de Estudios sobre Sistemas Educativos/ International Journal of Studies in Educational Systems. 2014; 2(4): 54. 
${ }^{6}$ «La vejez, como parte del ciclo vital, es inseparable de otras etapas y experiencias de la vida, por lo que existe una franca diversidad en la forma como envejecen las personas, desde las sanas y productivas hasta las otras gravemente enfermas e inactivas». (Morales Virgen, Juan José. (2004) Retos de la investigación gerontológica. En Geriatría. Mc Graw-Hill, México, p. 339.

${ }^{7}$ Véase un resumen en: BRAILOVSKY, S. RodRíGUEZ, R. (2004). Teorías sobre el envejecimiento. En Geriatría. Mc Graw Hill, México. pp. 25-27.

${ }^{8}$ De LeÓn, Alvaro. (2013). El abordaje interdisciplinar en la vejez y el envejecimiento con riesgo social. Un estudio de caso en la Cátedra de Geriatría del Hospital de Clínicas (tesis). Montevideo: Universidad de la República de Uruguay. Consultado el 3 de diciembre de 2018. Disponible en: file:///D:/2018/Gerontología\% 20e\%20interdisciplina/El\%20abordaje\%20interdisciplinar\%20en\%20la\%20vejez..pdf

${ }^{9}$ Op. Cit., pp. 59-60.

${ }^{10}$ Esta idea surge en otras descripciones de la interdisciplina y la gerontología: «Varios otros adjetivos precisan el modo de esta operación de la interdisciplinariedad. Es normativa, y también restrictiva, porque exige el establecimiento de normas que determinen la contribución y la conducta o desempeño de las ciencias, profesiones, especialidades y enfoques, que intervienen para tratar en torno al estudio y solución del problema dado y que, a la vez, restrinjan sus aportes y aciertos a sólo lo necesario y suficiente. En suma, que la interdisciplinariedad compuesta es acción convergente, y no simple acomodo de paralelas y yuxtapuestas visiones de un problema» (Borrero Cabal, Alfonso. En busca de la interdisciplinariedad: interdisciplinariedad y gerontologia. Consultado el 2 de diciembre de 2018. Disponible en: https://www.gerontologia.org/portal/information/ showlnformation.php?idinfo=862, s.p.

${ }^{11}$ LASSEN JUUL, ASKE. Biopolíticas de la vejez. Cómo el conocimiento sobre el envejecimiento forma políticas de envejecimiento active. Sociología histórica 5/ 2015: 342-343.

${ }^{12}$ SÁNCHEZ GonZÁleZ, DIEgo. Ambiente físico-social y envejecimiento de la población desde la gerontología ambiental y geografía. Implicaciones socioespaciales en América Latina. Revista de Geografía Norte Grande. 2015; 60: 97-114.

${ }^{13}$ «En el centro de la complejidad que caracteriza el mundo actual, la exploración de estrategias que accedan al alcance del bienestar social de la población nos conduce y motiva a un debate y reflexión desde los distintos estamentos políticos, académicos, técnicos. Pero acordamos en que la problemática será superada con políticas públicas integrales que contemplen la solidaridad y la participación de los mayores, de los profesionales, de los equipos de atención en tanto participen en la toma de decisions» (CABERO, LAURA ADRIANA. Una reflexión sobre la intervención social en la expresión actual de la vejez. Margen. 2017; 87: p. 5. Consultado el 4 de diciembre de 2018. Disponible en: https://www.margen.org/suscri/ margen87/cabero_87.pdf).

Medicina y Ética - Octubre-Diciembre 2019 - Vol. 30 - Núm. 4 


\section{J. E. Gómez Álvarez}

${ }^{14}$ OMS (2001). Campaña de la OMS por un envejecimiento activo p. 2. Consultado el 2 de diciembre de 2018. Disponible en:https://www.who.int/ageing/publications/alc_elmanual.pdf?ua $=1$

${ }^{15}$ Rodríguez García, Rosalía. Mendoza Martínez, Lilia. Calidad de Vida. En Geriatría. Mc Graw-Hill, México, 2004.

${ }^{16}$ Cf. LASSEN JUUL, ASKE. Biopolíticas de la vejez. Cómo el conocimiento sobre el envejecimiento forma políticas de envejecimiento active. Sociología Histórica 5/ 2015. 